\title{
Character association studies in Bread wheat genotypes for early heat tolerance and grain micronutrient content
}

\author{
Rabiya Parveen ${ }^{1}$, Satish Kumar Singh ${ }^{2}$, Mithilesh Kumar Singh ${ }^{2}$, Mainak \\ $\operatorname{Barman}^{3}$ 凶
}

\begin{abstract}
Thirty bread wheat genotypes were tested by adopting Randomized Block Design with three replications during 2019-20, under two different environments, i.e. early and timely sown conditions. The ultimate goal was to determine the relationship between yield and yield contributing traits and classify traits that have the highest direct and indirect effects on grain yield under both environmental circumstances. Grain yield was positively and significantly correlated with no. of tiller/plant, no. of grains/spike, relative water content, spike fertility, harvest index, grain zinc content and chlorophyll content at the level of both genotype and phenotype; conversely, found significantly negative with canopy temperature and non-significant with days to $50 \%$ flowering and seedling length. Under stress condition, the correlation for grain yield/plant was found significant, and it was correlated positively with no. of tiller/plant, spike fertility, relative water content, harvest index and grain zinc content and non-significantly with seedling length, no. of grain/spike, chlorophyll content, flag leaf area and thousand-grain weight. At the level of both genotype and phenotype, grain yield per plant had a high direct effect in the positive direction by harvest index followed by no. of tiller/plant, no. of grain/spike, relative water content. Thus, more weightage should be given towards these traits as selecting these traits can be rewarding to achieve high yielding wheat genotypes.
\end{abstract}

Key words: Bread wheat, Correlation coefficient analysis, Path coefficient analysis, Randomized Block Design

\section{Introduction}

Wheat (Triticum spp.) is counted among the most commonly grown cereal crops to assure the necessity for more than half of their protein and calorie consumption by one-third of the global population. Its contribution from the nutritional aspect is much higher than any other food crop, holding the first rank in area coverage in addition to second in total production following maize (FAOSTAT, 2019). Heat stress is one of the key abiotic limitations that hinder the production as well as productivity of wheat at some stages in germination and grain filling as the crop is adapted to cultivate in regions with a cooler climate. Systems of wheat breeding also require incorporating the production of genotypes holding

\section{Author's Address}

${ }^{1}$ Department of Plant Breeding and Genetics, Bihar agricultural University, Sabour, Bhagalpur, Bihar, India

${ }^{2}$ Department of Plant Breeding and Genetics, Dr. Rajendra Prasad Central Agricultural University, Pusa, Samastipur, Bihar, India

${ }^{3}$ Department of Genetics and Plant Breeding, Bidhan Chandra Krishi Viswavidyalaya, Mohanpur, Nadia, West Bengal, India E-mail.: mainakbarman4@gmail.com heat-resistance capacity as a key objective in addition to the common objective of enhancing grain yield. Joshi et al. (2007) suggested nurturing early-maturing genotypes of wheat that enables the genotypes to escape the stress, especially in eastern Gangetic plains where the crops are recorded with high terminal heat stress. Malnutrition of micronutrient, predominantly the deficiency in Zinc worldwide afflicts more than three billion people. The deficiency of Zinc is a decisive micronutrient deficit in human health that concerns more than one-third of the world's population (Stein et al., 2010). High-yielding cereal cultivars have contributed to the high incidence of $\mathrm{Zn}$ deficiency in humans by reducing $\mathrm{Zn}$ concentration in grain by dilution and erosion in soils (Cakmak et al., 2008). Studies on correlation and path analysis assist to elucidate the relationship between different traits and their direct as well as indirect effects on grain yield. We can determine the degree and direction of the association between a range of yield contributing characters and yield with the help of correlation analysis. The path coefficients facilitate 
analyzing the direct and indirect effect of an independent trait on a dependent one i.e yield. Plant breeders have used the method of analyzing the path coefficients to assist categorizing advantageous traits for selection to boost crop yield. The present study of correlation and path coefficient analysis was carried out with the intention of digging out the suitable traits to achieve early heat tolerance and higher grain zinc content in bread wheat by analyzing grain yield and its attributing characters.

\section{Materials and Methods}

The current analysis was conducted at the experimental plot of wheat breeding section of Dr. Rajendra Prasad Central Agricultural University, Pusa, Bihar, India during Rabi 2019-20. The materials included in the experiment consisted of 30 hexaploid bread wheat (Triticum aestivum L.) genotypes counting variety HD-2967 which was used as a check for grain yield under normal and early sowing conditions along with WB-02, which was used as a check for grain zinc content. Two different climatic conditions were considered to nurture selected 30 genotypes, i.e., early and timely sown. The experiment was conducted in Randomized Block Design (RBD) and the number of replication included was 3 in each setting. In each replication, all the genotypes were sown in a plot divided into number of rows (5) of $3 \mathrm{~m}$ separated by a distance of $20 \mathrm{~cm}$ under both early and timely sown conditions. Irrigation was provided in the field at regular intervals based on rainfall pattern and suggested agronomic and traditional cultural practices were adopted to achieve healthy and improved crops. Observations were recorded for 17 quantitative traits viz., plant height, seedling length, canopy temperature, tiller/plant, ear length, flag leaf area, relative water content, days to $50 \%$ flowering, no. of grain/spike, chlorophyll content, spike fertility, test weight, days to maturity, harvest index, grain yield/plant, grain zinc content and heat susceptibility index. Grain zinc content was estimated at ICRISAT, HP-MAL, Hyderabad, India, with the help of an X-ray Fluorescence Spectrophotometer (XRF) (Barman et al., 2020a). WINDOSTAT version 7.0 was used for statistical analysis of the recorded data on various traits. Calculation of phenotypic as well as genotypic correlation coefficients was performed from the components of variance and covariance following Barman et al. (2020b). The correlation coefficient of yield with different quantitative traits was divided into direct as well as indirect effects by analyzing path coefficient as per the method followed by Baye et al. (2020).

\section{Genotypic correlation coefficient}

Considering genotypic values $\left(\sigma^{2}\right.$ gi and $\sigma^{2}$ gj) the genotypic correlation were calculated:

$$
r\left(g_{i}, g_{j}\right)=\frac{\operatorname{Cov}\left(g_{i}, g_{j}\right)}{\sqrt{V\left(g_{i}\right) \cdot V\left(g_{j}\right)}}
$$

Phenotypic correlation coefficient

$$
r\left(p_{i}, p_{j}\right)=\frac{\operatorname{Cov}\left(p_{i}, p_{j}\right)}{\sqrt{V\left(P_{i}\right) \cdot V\left(p_{j}\right)}}
$$

\section{Results and Discussion}

Character association under timely sown condition

At the phenotypic level (Table 1), Grain yield/plant was positively and significantly correlated with harvest index (0.6215), relative water content (0.5074), number of grain per spike (0.4596), no. of tiller/plant (0.4288), chlorophyll content (0.3913), spike fertility (0.4528) and grain $\mathrm{Zn}$ content (0.3523). A significant negative association was found for canopy temperature $(-0.6445)$. These findings were alike with outcomes as obtained by Khan et al. (2010) and Dogan (2009) (Table 1). The association for grain yield per plant was found significant, and it was associated positively with tiller/plant, no. of grain/spike and harvest index by Kotal et al. (2010). Bahar et al. (2011) and Barman et al. (2020b) found that grain yield was associated negatively with canopy temperature, positively with test weight, ear length, no. of grain/spike and tiller/plant and for days to $50 \%$ flowering. A significant correlation was found with ear length as well as harvest index as earlier reported by Mohsen et al. (2012) and Zafarnaderi et al. (2013). Grain yield was associated positively with the grain zinc content. Sharma et al. (2018) found a significant association between mean iron $(\mathrm{Fe})$ values with days to maturity and grain yield/plant, and the mean zinc value ( $\mathrm{Zn}$ ) was also found to be correlated with grain yield/plant.

Character association under early sown condition At the phenotypic level (Table 2), grain yield per plant was found significantly and positively 
Character association studies in Bread wheat genotypes

Table 1: Phenotypic and Genotypic correlation coefficient for 16 traits in hexaploid wheat under timely-sown condition

\begin{tabular}{|c|c|c|c|c|c|c|c|c|c|c|c|c|c|c|c|c|}
\hline Characters & & PH & SL & TPP & FLA & RWC & DFF & CT & EL & GPS & SF & CHL & TGW & DM & HI & $\mathbf{Z n}$ \\
\hline \multirow{2}{*}{ SL } & $\mathbf{P}$ & -0.0690 & & & & & & & & & & & & & & \\
\hline & $\mathbf{G}$ & -0.5048 & & & & & & & & & & & & & & \\
\hline \multirow{2}{*}{ TPP } & $\mathbf{P}$ & 0.0703 & -0.0093 & & & & & & & & & & & & & \\
\hline & $\mathbf{G}$ & 0.2435 & -0.0279 & & & & & & & & & & & & & \\
\hline \multirow{2}{*}{ FLA } & $\mathbf{P}$ & 0.0219 & $0.3126^{* *}$ & $0.3702 * *$ & & & & & & & & & & & & \\
\hline & $\mathbf{G}$ & 0.0268 & 0.3371 & 0.4146 & & & & & & & & & & & & \\
\hline \multirow{2}{*}{ RWC } & $\mathbf{P}$ & 0.0002 & 0.0398 & $0.3051 * *$ & 0.0930 & & & & & & & & & & & \\
\hline & $\mathbf{G}$ & 0.0274 & 0.0690 & 0.7708 & 0.2695 & & & & & & & & & & & \\
\hline \multirow{2}{*}{ DFF } & $\mathbf{P}$ & 0.0514 & 0.2424 * & 0.0025 & -0.0506 & 0.0854 & & & & & & & & & & \\
\hline & $\mathbf{G}$ & -0.0899 & 0.3125 & -0.0028 & -0.0489 & 0.2695 & & & & & & & & & & \\
\hline \multirow{2}{*}{ CT } & $\mathbf{P}$ & $-0.2287^{*}$ & -0.0142 & $-0.2377 *$ & -0.2017 & $-0.4086 * *$ & -0.2005 & & & & & & & & & \\
\hline & $\mathbf{G}$ & -0.4534 & 0.0083 & -0.3149 & -0.2305 & -0.9036 & -0.2649 & & & & & & & & & \\
\hline \multirow{2}{*}{ EL } & $\mathbf{P}$ & -0.0085 & 0.0265 & 0.0252 & $0.2160 *$ & -0.0454 & -0.0695 & -0.0158 & & & & & & & & \\
\hline & $\mathbf{G}$ & -0.0091 & -0.0043 & 0.0403 & 0.2842 & 0.2097 & -0.1928 & 0.1435 & & & & & & & & \\
\hline \multirow{2}{*}{ GPS } & $\mathbf{P}$ & 0.0937 & -0.1063 & 0.1745 & -0.0100 & $0.3934^{* *}$ & 0.0710 & $-0.5614 * *$ & -0.0636 & & & & & & & \\
\hline & $\mathbf{G}$ & 0.5793 & -0.1491 & 0.1436 & -0.0365 & 0.9189 & 0.1470 & -0.8789 & -0.1580 & & & & & & & \\
\hline \multirow{2}{*}{ SF } & $\mathbf{P}$ & 0.1976 & 0.0469 & $0.2711 * *$ & $0.2217 *$ & $0.4993^{* *}$ & 0.1934 & $-0.6569 * *$ & 0.0584 & $0.5706^{* *}$ & & & & & & \\
\hline & $\mathbf{G}$ & 0.5355 & 0.0455 & 0.3571 & 0.3008 & 0.9046 & 0.2501 & -0.9236 & -0.0828 & 0.7982 & & & & & & \\
\hline \multirow{2}{*}{ CHL } & $\mathbf{P}$ & 0.0855 & -0.0138 & 0.0914 & 0.0808 & $0.4635 * *$ & -0.0056 & $-0.5214 * *$ & 0.0662 & $0.5147 * *$ & $0.6070 * *$ & & & & & \\
\hline & $\mathbf{G}$ & 0.9090 & -0.1053 & 0.0733 & 0.3379 & 0.9435 & 0.1325 & -0.9896 & -0.2243 & 0.9070 & 0.9441 & & & & & \\
\hline \multirow{2}{*}{ TGW } & $\mathbf{P}$ & \begin{tabular}{|l|}
0.1043 \\
\end{tabular} & -0.0654 & 0.0321 & $0.2093 *$ & -0.0134 & 0.1018 & -0.0926 & 0.0753 & 0.0454 & 0.1478 & 0.1364 & & & & \\
\hline & $\mathbf{G}$ & 0.1773 & -0.0617 & 0.0366 & 0.2733 & -0.0380 & 0.1487 & 0.0143 & 0.1185 & 0.1206 & 0.1663 & 0.0184 & & & & \\
\hline \multirow{2}{*}{ DM } & $\mathbf{P}$ & 0.1182 & 0.3287 ** & 0.0470 & -0.0272 & 0.1433 & $0.8027^{* * *}$ & $-0.3131 * *$ & -0.0765 & 0.1710 & 0.2094 * & 0.0643 & 0.0309 & & & \\
\hline & $\mathbf{G}$ & -0.0969 & 0.3892 & 0.0642 & -0.0151 & 0.5850 & 0.9516 & -0.3707 & -0.2109 & 0.2758 & 0.3967 & 0.5343 & 0.0229 & & & \\
\hline \multirow{2}{*}{ HI } & $\mathbf{P}$ & 0.1661 & -0.0635 & $0.2288 *$ & 0.1998 & $0.4808 * *$ & 0.1470 & $-0.7388 * *$ & -0.0636 & $0.4175^{* *}$ & $0.7602 * *$ & $0.5728^{* *}$ & 0.0579 & 0.1663 & & \\
\hline & $\mathbf{G}$ & 0.5711 & -0.0639 & 0.3137 & 0.2656 & 0.9250 & 0.1812 & -0.9801 & -0.1542 & 0.7060 & 0.8952 & 0.9625 & 0.0132 & 0.3207 & & \\
\hline \multirow{2}{*}{$\mathbf{Z n}$} & $\mathbf{P}$ & 0.0667 & 0.0797 & $0.2527 *$ & $0.3894 * *$ & 0.1521 & 0.1394 & $-0.3210 * *$ & 0.0380 & 0.0345 & $0.2841 * *$ & 0.0508 & 0.2467 * & 0.1057 & $0.3541 * *$ & \\
\hline & $\mathbf{G}$ & 0.2439 & 0.0773 & 0.2787 & 0.4094 & 0.3816 & 0.1673 & -0.3887 & 0.0557 & 0.0651 & 0.3507 & 0.1977 & 0.2739 & 0.1308 & 0.4588 & \\
\hline \multirow{2}{*}{ GY } & $\mathbf{P}$ & 0.0464 & -0.0740 & $0.4288^{* *}$ & 0.1718 & $0.5074^{* *}$ & -0.0091 & $-0.6445^{* *}$ & 0.0325 & $0.4596^{* *}$ & $0.4528^{* *}$ & $0.3913^{* *}$ & 0.0012 & 0.0341 & $0.6215^{* *}$ & $0.3523^{* *}$ \\
\hline & $\mathbf{G}$ & 0.2876 & -0.0992 & 0.5732 & 0.1891 & 0.9704 & -0.0008 & -0.8822 & 0.1384 & 0.6219 & 0.7649 & 0.9720 & -0.0112 & 0.0881 & 0.8830 & 0.4604 \\
\hline
\end{tabular}

* * Significance at $1 \%$ level $=\mathbf{0 . 2 7 0 2}$

* Significance at $5 \%=0.2072$ 
Rabiya et al.

Table 2: Phenotypic and Ghenotypic correlation coefficient for 16 traits in hexaploid wheat under early-sown condition

\begin{tabular}{|c|c|c|c|c|c|c|c|c|c|c|c|c|c|c|c|c|}
\hline Characters & & PH & SL & TPP & FLA & RWC & DFF & CT & $\mathbf{E L}$ & GPS & SF & CHL & TGW & DM & HI & $\mathbf{Z n}$ \\
\hline \multirow{2}{*}{ SL } & $\mathbf{P}$ & 0.1297 & & & & & & & & & & & & & & \\
\hline & $\mathbf{G}$ & 0.4976 & & & & & & & & & & & & & & \\
\hline \multirow{2}{*}{ TPP } & $\mathbf{P}$ & 0.0297 & $\begin{array}{l}-0.1720 \\
\end{array}$ & & & & & & & & & & & & & \\
\hline & G & -0.0826 & -0.1966 & & & & & & & & & & & & & \\
\hline \multirow{2}{*}{ FLA } & $\mathbf{P}$ & 0.0470 & $-0.2729 * *$ & 0.0386 & & & & & & & & & & & & \\
\hline & G & -0.0026 & -0.3199 & 0.0467 & & & & & & & & & & & & \\
\hline \multirow{2}{*}{ RWC } & $\mathbf{P}$ & -0.1602 & 0.0920 & 0.0835 & $0.2574 *$ & & & & & & & & & & & \\
\hline & $\mathbf{G}$ & 0.9241 & 0.1511 & 0.3618 & 0.8723 & & & & & & & & & & & \\
\hline \multirow{2}{*}{ DFF } & $\mathbf{P}$ & 0.1465 & $-0.2263 *$ & -0.0632 & -0.0843 & $-0.2086 *$ & & & & & & & & & & \\
\hline & $\mathbf{G}$ & 0.4275 & -0.2474 & -0.0492 & -0.1236 & -0.5967 & & & & & & & & & & \\
\hline \multirow{2}{*}{ CT } & $\mathbf{P}$ & -0.1092 & -0.1853 & -0.1524 & -0.0990 & -0.2407 * & $0.2956 * *$ & & & & & & & & & \\
\hline & $\mathbf{G}$ & -0.1213 & -0.2758 & $\begin{array}{l}-0.1675 \\
\end{array}$ & -0.1018 & -0.7362 & 0.5379 & & & & & & & & & \\
\hline \multirow{2}{*}{ EL } & $\mathbf{P}$ & 0.1002 & 0.1076 & 0.0610 & -0.0026 & 0.1053 & -0.1670 & -0.0148 & & & & & & & & \\
\hline & $\mathbf{G}$ & -0.1796 & 0.1233 & 0.0660 & 0.0198 & 0.5194 & -0.2492 & 0.0605 & & & & & & & & \\
\hline \multirow{2}{*}{ GPS } & $\mathbf{P}$ & 0.1504 & 0.1098 & 0.0695 & 0.1331 & $0.4344 * *$ & -0.1057 & $-0.3968^{* *}$ & -0.0866 & & & & & & & \\
\hline & $\mathbf{G}$ & 0.5180 & 0.4326 & 0.1346 & 0.5823 & 0.9383 & -0.5405 & -0.4477 & -0.3107 & & & & & & & \\
\hline \multirow{2}{*}{ SF } & $\mathbf{P}$ & 0.0476 & 0.0775 & $0.2350 *$ & $0.2086 *$ & $0.2776 * *$ & -0.0225 & -0.1764 & 0.0617 & $0.2417 *$ & & & & & & \\
\hline & $\mathbf{G}$ & 0.2536 & 0.1281 & 0.4950 & 0.4110 & 0.9446 & -0.0419 & -0.4639 & 0.2182 & 0.9342 & & & & & & \\
\hline \multirow{2}{*}{ CHL } & $\mathbf{P}$ & 0.0657 & 0.1803 & 0.1293 & -0.0259 & $0.2325 *$ & 0.0405 & $-0.2526 *$ & -0.0395 & 0.3230 ** & 0.1537 & & & & & \\
\hline & $\mathbf{G}$ & 0.0093 & 0.2777 & 0.2411 & 0.0347 & 0.4427 & 0.1228 & -0.2973 & -0.2056 & -0.0726 & 0.5600 & & & & & \\
\hline \multirow{2}{*}{ TGW } & $\mathbf{P}$ & 0.0685 & -0.0895 & -0.0131 & $0.2789 * *$ & 0.1783 & -0.1548 & $-0.2559 *$ & 0.0357 & 0.1547 & 0.0679 & 0.1589 & & & & \\
\hline & G & 0.1402 & -0.1332 & -0.0281 & 0.3385 & 0.5044 & -0.1450 & -0.3815 & -0.1558 & 0.5673 & 0.2606 & 0.2207 & & & & \\
\hline \multirow{2}{*}{ DM } & $\mathbf{P}$ & 0.1055 & $-0.2231 *$ & $\begin{array}{l}-0.0632 \\
\end{array}$ & -0.1249 & $-0.2350 *$ & $0.8559^{* *}$ & $0.4100^{* *}$ & -0.1761 & -0.1325 & -0.0778 & 0.0181 & -0.0938 & & & \\
\hline & $\mathbf{G}$ & 0.6926 & -0.2416 & -0.0205 & -0.1858 & -0.7112 & 0.9078 & 0.5552 & -0.4242 & -0.3712 & -0.0984 & 0.0849 & -0.1595 & & & \\
\hline \multirow{2}{*}{ HI } & $\mathbf{P}$ & 0.0992 & $0.2382 *$ & 0.1361 & 0.0699 & 0.3012 ** & -0.1358 & $-0.4275^{* *}$ & 0.0234 & 0.1486 & 0.2084 * & $0.3193 * *$ & $0.3598^{* *}$ & -0.1357 & & \\
\hline & $\mathbf{G}$ & 0.5070 & 0.2366 & 0.1977 & 0.1449 & 0.8203 & -0.2606 & -0.6451 & -0.2985 & 0.5318 & 0.5544 & 0.6320 & 0.4270 & -0.1981 & & \\
\hline \multirow{2}{*}{$\mathbf{Z n}$} & $\mathbf{P}$ & 0.0283 & $-0.2866 * *$ & 0.1612 & $0.4169 * *$ & 0.1547 & 0 & $-0.2848 * *$ & -0.1015 & 0.0756 & 0.2204 * & 0.1007 & $0.3201 * *$ & -0.0363 & $0.4119 * *$ & \\
\hline & $\mathbf{G}$ & 0.0726 & -0.3091 & 0.1805 & 0.4591 & 0.4990 & 0.0030 & -0.4205 & -0.2450 & 0.1689 & 0.3468 & 0.1797 & 0.3304 & -0.0620 & 0.5073 & \\
\hline \multirow{2}{*}{ GY } & $\mathbf{P}$ & -0.0560 & 0.1589 & $0.2074^{*}$ & 0.1096 & $0.2960 * *$ & -0.0845 & -0.1521 & -0.0900 & 0.1604 & $0.3743^{* *}$ & 0.1105 & 0.0530 & -0.1377 & $0.3038^{* * *}$ & $0.2229^{* * *}$ \\
\hline & $\mathbf{G}$ & 0.6162 & 0.2923 & 0.3055 & 0.6299 & 0.6044 & 0.6953 & -0.3395 & 0.3347 & 0.1342 & 0.8786 & 0.5601 & 0.5789 & 0.6780 & 0.9930 & 0.2259 \\
\hline
\end{tabular}

$*$ * Significance at $1 \%$ level $=0.2702$

$*$ Significance at $\mathbf{5 \%}=\mathbf{0 . 2 0 7 2}$ 
correlated with spike fertility (0.3743), relative water content (0.2960), harvest index (0.3038), grain zinc content $(0.2229)$ and no. of tiller/plant (0.2074). At the genotypic level (Table 2), the correlation for grain yield/plant was found positive with ear length (0.3347), harvest index (0.9930), spike fertility (0.8786), relative water content (0.6044), flag leaf area (0.6299), plant height (0.6162), days to maturity $(0.6780)$, test weight (0.5789), days to 50 per cent flowering (0.6953), chlorophyll content $(0.5601)$, no. of tillers/plant (0.3055), seedling length (0.2923), grain zinc content $(0.2259)$ and no. of grains per spike $(0.1342)$. On the other hand, grain yield was recorded to be negatively correlated with canopy temperature (-0.3395). Similar findings were also obtained earlier by many other researchers like Bahar et al. (2011) found significant negative associations between canopy temperature and grain yield, Iftikhar et al. (2012) found a significant positive correlation for grain yield with number of grains/spike, test weight as well as flag leaf area, Mohsen et al. (2012) found positive association for spike length, test weight, number of spikelet/spike, number of tillers/plant and number of grain/spike with grain yield/plant. Grain yield/ha was reported to be positively associated with harvest index by Chimdesa et al. (2017).

\section{Path coefficient analysis under timely sown condition}

At both genotypic and phenotypic levels (Table 3 and 4), grain yield per plant had a high direct effect in the positive direction by harvest index. High direct effect but in negative direction was found at the level of genotype by days to maturity, whereas, at the level of phenotype, canopy temperature and spike fertility exhibited high direct effect in the negative direction on grain yield. The number of grains/spike, biological yield per plant and harvest index displayed a direct and positive effect on grain yield as reported earlier by Kumar et al. (2014). Significantly positive and direct effect was perceived for the dependent variable i.e., yield/plant for no. of grains/spike followed by no. of effective tillers/plant and seed index which was in accordance with Sharma et al. (2018). Verma et al. (2019) reported a direct and significant positive effect on yield/plant by biological yield/plant followed by harvest index, days to $50 \%$ flowering, test weight and tillers/plant.

\section{Phenotypic Path Coefficient}

A strong positive phenotypic correlation of harvest index with grain yield $(0.6215)$ resulted due to its direct positive effect on grain yield $(0.4025)$ and a considerable positive indirect effect via number of grain per spike (0.0908), canopy temperature (0.2701), and grain zinc content (0.0531). Positive phenotypic association (0.4596) for no. of grains per spike was perceived with grain yield, which was attributed to its direct positive effect $(0.2174)$ and a considerable indirect effect via canopy temperature $(0.2053)$, no. of tiller/plant $(0.0454)$ and harvest index (0.1680). Positive phenotypic correlation of plant height with grain yield/plant (0.0464) was although negated by its direct effect (0.0518). However, it is due to its cumulative indirect effects via canopy temperature (0.0836), no. of tiller/plant $(0.0183)$, harvest index $(0.0669)$ on grain yield/plant. The seedling length was correlated negatively with grain yield/plant (0.0740 ), which was ascribed by additions of its indirect effect via days to maturity $(-0.0591)$, flag leaf area $(-0.0343)$, no. of grains per spike ($0.0231)$, harvest index $(-0.0256)$, despite having a positive direct effect $(0.0512)$ on grain yield. Phenotypic positive association of no. of tiller per plant $(0.4288)$ on grain yield/plant was contributed by its direct effect (0.2601), which further increased by its positive indirect effect via canopy temperature (0.0869), no. of grains per spike (0.0379), relative water content $(0.0618)$, harvest index (0.0921). Although flag leaf area exhibited a negative direct effect $(-0.1096)$ on grain yield, the positive phenotypic correlation of flag leaf area (0.1718) on grain yield/plant was due to cumulative indirect positive effect via no. of tiller per plant (0.0963), canopy temperature (0.0737), harvest index (0.0804), and grain zinc content (0.0584). Positive phenotypic correlation of relative water content on grain yield (0.5074) was attributed due to direct positive effect of relative water content (0.2026), which was further increased by its cumulative indirect effect in positive direction via canopy temperature (0.1494), number of grain per spike (0.0855), and harvest index (0.1935). A negative correlation of days to $50 \%$ flowering (0.0091) with grain yield/plant resulted from its cumulative indirect effect via spike fertility ($0.0665)$, days to maturity $(-0.1443)$. It displayed a direct positive effect $(0.0087)$ on grain yield. Grain

115

Environment Conservation Journal 


\section{Rabiya et al.}

Table 3: Analysis of Phenotypic path coefficient of fifteen traits on grain yield in hexaploid wheat in timely-sown condition

\begin{tabular}{|c|c|c|c|c|c|c|c|c|c|c|c|c|c|c|c|}
\hline Characters & PH & SL & TPP & FLA & R WC & DFF & CT & EL & GPS & SF & CHL & TGW & DM & HI & $\mathrm{ZnC}$ \\
\hline PH & -0.0518 & 0.0036 & -0.0036 & -0.0011 & 0.0001 & -0.0027 & 0.0119 & 0.0004 & -0.0049 & -0.0102 & -0.0044 & -0.0054 & -0.0061 & -0.0086 & -0.0035 \\
\hline SL & -0.0035 & 0.0512 & -0.0005 & 0.016 & 0.002 & 0.0124 & -0.0007 & 0.0014 & -0.0054 & 0.0024 & -0.0007 & -0.0033 & 0.0168 & -0.0033 & 0.0041 \\
\hline TPP & 0.0183 & -0.0024 & 0.2601 & 0.0963 & 0.0793 & 0.0006 & -0.0618 & 0.0066 & 0.0454 & 0.0705 & 0.0238 & 0.0083 & 0.0122 & 0.0595 & 0.0657 \\
\hline FLA & -0.0024 & -0.0343 & -0.0406 & -0.1096 & -0.0102 & 0.0055 & 0.0221 & -0.0237 & 0.0011 & -0.0243 & -0.0089 & -0.0229 & 0.0030 & -0.0219 & -0.0427 \\
\hline RWC & 0.0001 & 0.0081 & 0.0618 & 0.0188 & 0.2026 & 0.0173 & -0.0828 & -0.0092 & 0.0797 & 0.1011 & 0.0939 & -0.0027 & 0.0290 & 0.0974 & 0.0308 \\
\hline DFF & 0.0004 & 0.0021 & 0.0001 & -0.0004 & 0.0007 & 0.0087 & -0.0018 & -0.0006 & 0.0006 & 0.0017 & 0.0001 & 0.0009 & 0.007 & 0.0013 & 0.0012 \\
\hline CT & 0.0836 & 0.0052 & 0.0869 & 0.0737 & 0.1494 & 0.0733 & -0.3656 & 0.0058 & 0.2053 & 0.2402 & 0.1906 & 0.0338 & 0.1145 & 0.2701 & 0.1174 \\
\hline EL & -0.0008 & 0.0025 & 0.0024 & 0.0207 & -0.0044 & -0.0067 & -0.0015 & 0.0960 & -0.0061 & 0.0056 & 0.0064 & 0.0072 & -0.0074 & -0.0061 & 0.0037 \\
\hline GPS & 0.0204 & -0.0231 & 0.0379 & -0.0022 & 0.0855 & 0.0154 & -0.1220 & -0.0138 & 0.2174 & 0.1240 & 0.1119 & 0.0099 & 0.0372 & 0.0908 & 0.0075 \\
\hline $\mathbf{S F}$ & -0.0679 & -0.0161 & -0.0932 & -0.0763 & -0.1717 & -0.0665 & 0.2259 & -0.0201 & -0.1963 & -0.3439 & -0.2088 & -0.0508 & -0.0720 & -0.2614 & -0.0977 \\
\hline CHL & -0.0031 & 0.0005 & -0.0033 & -0.0029 & -0.0167 & 0.0002 & 0.0187 & -0.0024 & -0.0185 & -0.0218 & -0.0359 & -0.0049 & -0.0023 & -0.0206 & -0.0018 \\
\hline TGW & -0.0025 & 0.0015 & -0.0008 & -0.0049 & 0.0003 & -0.0024 & 0.0022 & -0.0018 & -0.0011 & -0.0035 & -0.0032 & -0.0236 & -0.0007 & -0.0014 & -0.0058 \\
\hline DM & -0.0213 & -0.0591 & -0.0084 & 0.0049 & -0.0258 & -0.1443 & 0.0563 & 0.0138 & -0.0308 & -0.0376 & -0.0116 & -0.0056 & -0.1798 & -0.0299 & -0.019 \\
\hline HI & 0.0669 & -0.0256 & 0.0921 & 0.0804 & 0.1935 & 0.0592 & -0.2973 & -0.0256 & 0.1680 & 0.3060 & 0.2305 & 0.0233 & 0.0669 & 0.4025 & 0.1425 \\
\hline $\mathrm{ZnC}$ & 0.0100 & 0.0119 & 0.0379 & 0.0584 & 0.0228 & 0.0209 & -0.0481 & 0.0057 & 0.0052 & 0.0426 & 0.0076 & 0.0370 & 0.0158 & 0.0531 & 0.1499 \\
\hline GY & 0.0464 & -0.0740 & 0.4288 & 0.1718 & 0.5074 & -0.0091 & -0.6445 & 0.0325 & 0.4596 & 0.4528 & 0.3913 & 0.0012 & 0.0341 & 0.6215 & 0.3523 \\
\hline
\end{tabular}


Character association studies in Bread wheat genotypes

Table 4: Analysis of Genotypic path coefficient of fifteen traits on grain yield in hexaploid wheat in timely-sown condition.

\begin{tabular}{|c|c|c|c|c|c|c|c|c|c|c|c|c|c|c|c|}
\hline Characters & PH & SL & TPP & FLA & RWC & DFF & CT & $\mathbf{E L}$ & GPS & SF & CHL & TGW & DM & HI & $\mathrm{ZnC}$ \\
\hline PH & -0.1647 & 0.0832 & -0.0401 & -0.0044 & -0.0045 & 0.0148 & 0.0747 & 0.0148 & -0.0954 & -0.0882 & -0.1827 & -0.0292 & 0.0160 & -0.0941 & -0.0402 \\
\hline SL & -0.0233 & 0.0462 & -0.0013 & 0.0156 & 0.0032 & 0.0144 & 0.0004 & -0.0002 & -0.0069 & 0.0021 & -0.0049 & -0.0029 & 0.0180 & -0.0030 & 0.0036 \\
\hline TPP & 0.0622 & -0.0071 & 0.2553 & 0.1058 & 0.1968 & -0.0007 & -0.0804 & 0.0103 & 0.0367 & 0.0912 & 0.0187 & 0.0093 & 0.0164 & 0.0801 & 0.0712 \\
\hline FLA & -0.0064 & -0.0801 & -0.0986 & -0.2377 & -0.0641 & 0.0116 & 0.0548 & -0.0676 & 0.0087 & -0.0715 & -0.0803 & -0.0650 & 0.0036 & -0.0631 & -0.0973 \\
\hline RWC & 0.0043 & 0.0109 & 0.1215 & 0.0425 & 0.1577 & 0.0425 & -0.1898 & 0.0331 & 0.1449 & 0.1584 & 0.1803 & -0.0060 & 0.0922 & 0.1616 & 0.0602 \\
\hline DFF & -0.0152 & 0.0527 & -0.0005 & -0.0082 & 0.0454 & 0.1686 & -0.0447 & -0.0325 & 0.0248 & 0.0422 & 0.0223 & 0.0251 & 0.1604 & 0.0305 & 0.0282 \\
\hline CT & 0.2266 & -0.0041 & 0.1573 & 0.1152 & 0.4014 & 0.1324 & -0.4996 & -0.0717 & 0.4391 & 0.4615 & 0.6441 & -0.0071 & 0.1852 & 0.5397 & 0.1942 \\
\hline EL & -0.0175 & -0.0008 & 0.0078 & 0.0553 & 0.0408 & -0.0375 & 0.0279 & 0.1947 & -0.0308 & -0.0161 & -0.0437 & 0.0231 & -0.0411 & -0.0300 & 0.0108 \\
\hline GPS & 0.1115 & -0.0287 & 0.0277 & -0.0070 & 0.1769 & 0.0283 & -0.1692 & -0.0304 & 0.1926 & 0.1537 & 0.2902 & 0.0232 & 0.0531 & 0.1359 & 0.0125 \\
\hline SF & 0.0722 & 0.0061 & 0.0481 & 0.0405 & 0.1354 & 0.0337 & -0.1245 & -0.0112 & 0.1076 & 0.1348 & 0.1677 & 0.0224 & 0.0535 & 0.1206 & 0.0473 \\
\hline CHL & -0.2332 & 0.0221 & -0.0154 & -0.0710 & -0.2404 & -0.0278 & 0.3132 & 0.0472 & -0.3168 & -0.2616 & -0.2102 & -0.0039 & -0.1123 & -0.2444 & -0.0416 \\
\hline TGW & -0.0048 & 0.0017 & -0.001 & -0.0074 & 0.001 & -0.0040 & -0.0004 & -0.0032 & -0.0033 & -0.0045 & -0.0005 & -0.0272 & -0.0006 & -0.0004 & -0.0074 \\
\hline DM & 0.0469 & -0.1884 & -0.0311 & 0.0073 & -0.2832 & -0.4607 & 0.1795 & 0.1021 & -0.1335 & -0.1920 & -0.2586 & -0.0111 & -0.4841 & -0.1552 & -0.0633 \\
\hline HI & 0.1992 & -0.0223 & 0.1094 & 0.0926 & 0.3574 & 0.0632 & -0.3766 & -0.0538 & 0.2462 & 0.3121 & 0.4054 & 0.0046 & 0.1118 & 0.3487 & 0.1600 \\
\hline $\mathrm{ZnC}$ & 0.0298 & 0.0094 & 0.0341 & 0.0500 & 0.0466 & 0.0204 & -0.0475 & 0.0068 & 0.0080 & 0.0428 & 0.0242 & 0.0335 & 0.0160 & 0.0561 & 0.1222 \\
\hline GY & 0.2876 & -0.0992 & 0.5732 & 0.1891 & 0.9704 & -0.0008 & -0.8822 & 0.1384 & 0.6219 & 0.7649 & 0.9720 & -0.0112 & 0.0881 & 0.8830 & 0.4604 \\
\hline
\end{tabular}




\section{Rabiya et al.}

yield/plant displayed negative phenotypic correlation (-0.6445) with canopy temperature resulted due to its direct negative effect $(-0.3656)$ and a considerable indirect effect via relative water content (-0.0828), no. of grain per spike $(-0.1220)$, harvest index (-0.2973). Low positive phenotypic correlation with grain yield per plant $(0.0325)$ with ear length resulted due to its direct positive effect (0.0960) and a substantial indirect effect via days to maturity (0.0138), canopy temperature (0.0058). A high positive phenotypic association of spike fertility with grain yield/plant $(0.4528)$ resulted due to indirect positive effect via relative water content (0.1011), canopy temperature (0.2402), number of grains per spike $(0.1240)$, harvest index $(0.3060)$, whereas, it exhibited negative direct effect (0.3439 ) on grain yield/plant. Grain yield/plant exhibited positive phenotypic correlation (0.3913) with Chlorophyll content and was ascribed to positive indirect effect via canopy temperature (0.1906), harvest index (0.2305), no. of grains/spike (0.1119), contrarily, it showed a direct negative effect on grain yield (0.0359). Thousand-grain weight exhibited a direct negative effect on grain yield (-0.0236). However, low positive phenotypic association with grain yield was attributed to positive indirect effect via harvest index (0.0233), grain zinc content (0.0370). Low positive phenotypic correlation of days to maturity with grain yield/plant $(0.0341)$ caused due to its positive indirect effect via canopy temperature $(0.1145)$, harvest index (0.0669), grain zinc content (0.0158), contrarily, on grain yield it showed negative direct effect (-0.1798). Grain zinc content exhibited a positive phenotypic association with grain yield (0.3523) caused by its positive direct effect $(0.1499)$ on grain yield/plant. A positive indirect effect was also depicted via harvest index (0.1425), canopy temperature $(0.1174)$, relative water content (0.0308), no. of tiller/plant (0.0657).

\section{Genotypic Path Coefficient}

Harvest index exhibited a strong positive genotypic correlation with grain yield $(0.8830)$ due to a strong positive direct effect on grain yield/plant (0.3487). It was also due to cumulative positive indirect effect via no. of tiller/plant (0.0801), canopy temperature (0.1942), spike fertility (0.0473), harvest index (0.1600). Positive phenotypic association of no. of tiller/plant caused due to its direct effect in a positive direction (0.2553) and from substantial indirect effect via canopy temperature (0.1573), relative water content (0.1215), no. of grains per spike (0.0277), spike fertility (0.0481) and harvest index (0.1094). Ear length resulted in a positive genotypic correlation with grain yield by positive direct effect $(0.1947)$ and also considerable positive indirect effect via the chlorophyll content (0.0472), relative water content (0.0331), plant height (0.0148), days to maturity (0.1021) and no. of tiller/plant (0.0103). A strong positive genotypic correlation of no. of grains per spike with grain yield/plant (0.6219) was build-up due to its positive direct effect on grain yield/plant $((0.1926)$ and involvement of its indirect effect via harvest index (0.2462), days to $50 \%$ flowering (0.0248), relative water content (0.1449), canopy temperature $(0.4391)$, number of tiller per plant (0.0367), spike fertility (0.1076), flag leaf area $(0.0087)$ and grain zinc content (0.0080). Negative genotypic association of seedling length was attributed to its indirect effect in negative direction via flag leaf area (-0.0801), no. of grains per spike $(-0.0287)$, days to maturity $(-0.1884)$, harvest index $(-0.0223)$ and number of tiller per plant $(-0.0071)$, contrarily, on grain yield / plant it showed a positive direct effect. Grain yield/plant (0.2876) was in positive genotypic correlation with Plant height resulted from its indirect positive effect via the number of tiller per plant $(0.0622)$, spike fertility $(0.0722)$, canopy temperature $(0.2266)$, harvest index (0.1992), days to maturity (0.0469), no. of grains per spike (0.1115), and grain zinc content (0.0298), contrarily, it was having a negative direct effect on grain yield (-0.1647). Positive genotypic association of flag leaf area ascribable to positive indirect effect via seedling length (0.0156), no. of tiller per plant (0.1058), relative water content (0.0425), ear length (0.0553), spike fertility (0.0405), canopy temperature (0.1152), harvest index (0.0926) and grain zinc content (0.0500) with grain yield (0.1891), whereas, it showed a negative direct effect on grain yield/plant (-0.2377). A strong genotypic correlation of relative water content with grain yield (0.9704) was ascribed to its direct effect $(0.1577)$ in a positive direction and also substantial indirect effect via no. of tiller/plant (0.1968), days to $50 \%$ flowering $(0.0454)$, canopy temperature (0.4014), ear length $(0.0408)$, no. of grains per spike (0.1769), spike fertility (0.1354), harvest 18

Environment Conservation Journal 
index (0.3574) and grain zinc content (0.0466). A negative correlation of days to $50 \%$ flowering with grain yield $(-0.0008)$ was ascribed to its indirect effect in a negative direction via ear length ($0.0375)$, chlorophyll content $(-0.0278)$, days to maturity $(-0.4607)$ and thousand-grain weight ($0.0040)$, contrarily, with positive direct effect $(0.1686)$ on grain yield/plant. Negative direct effect $(-0.4996)$ of Canopy temperature on grain yield/plant and its indirect effect in negative direction via no. of tiller per plant $(-0.0804)$, relative water content $(-0.1898)$, days to $50 \%$ flowering (-0.0447), no. of grains per spike ($0.1692)$, spike fertility $(-0.1245)$, harvest index ($0.3766)$ and grain zinc content $(-0.0475)$ establishes negative genotypic correlation $(-0.8822)$ with grain yield. Spike fertility showed a strong positive genotypic association with grain yield/plant (0.7649) resulted from its positive direct effect and also contributed by its positive indirect effect via days to $50 \%$ flowering (0.0422), no. of tiller/plant (0.0912), canopy temperature $(0.4615)$, no. of grains/spike (0.1537), relative water content (0.1584), HI (0.3121) and grain zinc content $(0.0428)$. Grain yield/plant $(-0.2102)$ had a negative direct effect by Chlorophyll content despite having a positive genotypic correlation (0.9720). The obtained result was ascribed to its indirect effect in a positive direction via spike fertility $(0.1677)$, Days to $50 \%$ flowering (0.0223), canopy temperature (0.6441), relative water content $(0.1803)$, no. of grains per spike $(0.2902)$, no. of tiller per plant (0.0187), harvest index (0.4054) and grain zinc content (0.0242). Negative genotypic correlation of thousand-grain weight on grain yield/plant $(-0.0112)$ resulted from its negative direct effect $(-0.0272)$ as well as from its indirect effect in negative direction via days to maturity ($0.0111)$, flag leaf area $(-0.0650)$, plant height ($0.0292)$ and canopy temperature $(-0.0071)$. The association for days to maturity was found positive with grain yield/plant (0.0881). This was contributed by its indirect positive effect via harvest index (0.1118), canopy temperature (0.1852), days to $50 \%$ flowering $(0.1604)$, relative water content (0.0922), no. of grains/spike (0.0531) and spike fertility (0.0535), contrarily, it had its direct effect $(-0.4841)$ on grain yield/plant in a negative direction. Grain zinc content exhibited positive genotypic correlation (0.4604) with grain yield/plant, resulting from its direct effect (0.1222) in a positive direction as well as the contribution of its substantial indirect effect via canopy temperature (0.1942), days to $50 \%$ flowering (0.0282), number of tiller per plant (0.0712), relative water content $(0.0602)$, spike fertility $(0.0473)$ and harvest index $(0.1600)$.

\section{Path coefficient analysis under early sown} condition

Table 5 and 6 , respectively, show the findings of the path coefficient analysis of the current experiment at both genotypic and phenotypic levels.

Analysis of path coefficient in stress condition displayed that at the level of genotype, on grain yield, most of the traits were having a high direct effect in the positive direction, and it was detected for chlorophyll content, plant height, relative water content, days to maturity and grain zinc content, whereas, at the level of phenotype, the moderate extent of direct effect was displayed by spike fertility. However, a high direct effect in a negative direction was perceived for flag leaf area, seedling length, harvest index and days to $50 \%$ flowering.

\section{Phenotypic Path Coefficient}

Spike fertility exhibited a positive direct effect $(0.2410)$ and an indirect effect via seedling length (0.0152), the number of tillers per plant (0.0373), days to maturity (0.0142), relative water content (0.0426), grain zinc content (0.0291) and harvest index (0.0331) leading to a positive association with grain yield $(0.3743)$. Under the early sown condition, seedling length showed a positive direct effect (0.1963) resulted in positive phenotypic association (0.1589) with grain yield/plant and also illustrated indirect effect in positive direction via harvest index (0.0378), relative water content (0.0141), days to maturity (0.0406) and spike fertility (0.0187). Positive association of no. of tiller per plant $(0.2074)$ with grain yield/plant was ascribed to its direct effect $(0.1589)$ in a positive direction as well as the endowment of its indirect effect via days to maturity $(0.0115)$, spike fertility (0.0566), harvest index (0.0216), relative water content $(0.0128)$ and grain zinc content $(0.0213)$. Harvest index had high and its indirect effect on grain yield/plant via seedling length (0.0467), days to maturity (0.0247), number of tiller per plant (0.0216), spike fertility $(0.0502)$, relative water content $(0.0462)$ and grain zinc content $(0.0544)$ 
Rabiya et al.

Table 5: Analysis of Phenotypic path coefficient of fifteen traits on grain yield in hexaploid wheat in early-sown condition

\begin{tabular}{|c|c|c|c|c|c|c|c|c|c|c|c|c|c|c|c|}
\hline Characters & PH & SL & TPP & FLA & RWC & DFF & CT & $\mathbf{E L}$ & GPS & SF & CHL & TGW & DM & HI & Zn \\
\hline PH & -0.0663 & -0.0086 & -0.0020 & -0.0031 & 0.0106 & -0.0097 & 0.0072 & -0.0066 & -0.0100 & -0.0032 & -0.0044 & -0.0045 & -0.0070 & -0.0066 & -0.0019 \\
\hline SL & 0.0255 & 0.1963 & -0.0338 & -0.0536 & 0.0181 & -0.0444 & -0.0364 & 0.0211 & 0.0215 & 0.0152 & 0.0354 & -0.0176 & -0.0438 & 0.0467 & -0.0563 \\
\hline TPP & 0.0047 & -0.0273 & 0.1589 & 0.0061 & 0.0133 & -0.0100 & -0.0242 & 0.0097 & 0.0110 & 0.0373 & 0.0205 & -0.0021 & -0.0100 & 0.0216 & 0.0256 \\
\hline FLA & 0.0003 & -0.0015 & 0.0002 & 0.0056 & 0.0014 & -0.0005 & -0.0006 & 0.0001 & 0.0007 & 0.0012 & -0.0001 & 0.0016 & -0.0007 & 0.0004 & 0.0023 \\
\hline RWC & -0.0246 & 0.0141 & 0.0128 & 0.0395 & 0.1533 & -0.0320 & -0.0369 & 0.0161 & 0.0666 & 0.0426 & 0.0356 & 0.0273 & -0.0360 & 0.0462 & 0.0237 \\
\hline DFF & 0.0207 & -0.032 & -0.0089 & -0.0119 & -0.0295 & 0.1413 & 0.0418 & -0.0236 & -0.0149 & -0.0032 & 0.0057 & -0.0219 & 0.1210 & -0.0192 & 0.0001 \\
\hline CT & -0.0114 & -0.0194 & -0.0159 & -0.0104 & -0.0252 & 0.0309 & 0.1045 & -0.0015 & -0.0415 & -0.0184 & -0.0264 & -0.0268 & 0.0429 & -0.0447 & -0.0298 \\
\hline EL & -0.0143 & -0.0153 & -0.0087 & 0.0004 & -0.0150 & 0.0238 & 0.0021 & -0.1422 & 0.0123 & -0.0088 & 0.0056 & -0.0051 & 0.0250 & -0.0033 & 0.0144 \\
\hline GPS & 0.0034 & 0.0025 & 0.0016 & 0.0030 & 0.0099 & -0.0024 & -0.0090 & -0.0020 & 0.0228 & 0.0055 & 0.0074 & 0.0035 & -0.0030 & 0.0034 & 0.0017 \\
\hline SF & 0.0115 & 0.0187 & 0.0566 & 0.0503 & 0.0669 & -0.0054 & -0.0425 & 0.0149 & 0.0582 & 0.2410 & 0.0370 & 0.0164 & -0.0188 & 0.0502 & 0.0531 \\
\hline CHL & -0.0041 & -0.0113 & -0.0081 & 0.0016 & -0.0145 & -0.0025 & 0.0158 & 0.0025 & -0.0202 & -0.0096 & -0.0626 & -0.0099 & -0.0011 & -0.0200 & -0.0063 \\
\hline TGW & -0.0017 & 0.0022 & 0.0003 & -0.0068 & -0.0043 & 0.0038 & 0.0062 & -0.0009 & -0.0038 & -0.0017 & -0.0039 & -0.0244 & 0.0023 & -0.0088 & -0.0078 \\
\hline DM & -0.0192 & 0.0406 & 0.0115 & 0.0227 & 0.0428 & -0.1559 & -0.0747 & 0.0321 & 0.0241 & 0.0142 & -0.0033 & 0.0171 & -0.1822 & 0.0247 & 0.0066 \\
\hline HI & 0.0158 & 0.0378 & 0.0216 & 0.0111 & 0.0478 & -0.0216 & -0.0679 & 0.0037 & 0.0236 & 0.0331 & 0.0507 & 0.0571 & -0.0215 & 0.1588 & 0.0654 \\
\hline $\mathrm{ZnC}$ & 0.0037 & -0.0379 & 0.0213 & 0.0551 & 0.0204 & 0.0001 & -0.0376 & -0.0134 & 0.0100 & 0.0291 & 0.0133 & 0.0423 & -0.0048 & 0.0544 & 0.1321 \\
\hline GY & -0.0560 & 0.1589 & 0.2074 & 0.1096 & 0.2960 & -0.0845 & -0.1521 & -0.0900 & 0.1604 & 0.3743 & 0.1105 & 0.0530 & -0.1377 & 0.3038 & 0.2229 \\
\hline
\end{tabular}


Character association studies in Bread wheat genotypes

Table 6: Analysis of Genotypic path coefficient analysis of fifteen traits on grain yield in hexaploid wheat in early-sown condition.

\begin{tabular}{|c|c|c|c|c|c|c|c|c|c|c|c|c|c|c|c|}
\hline Characters & PH & SL & TPP & FLA & RWC & DFF & CT & $\mathbf{E L}$ & GPS & SF & CHL & TGW & DM & HI & $\mathrm{ZnC}$ \\
\hline PH & 0.8284 & 0.7605 & -0.1262 & -0.004 & 1.5652 & 0.6533 & -0.1853 & -0.2745 & 0.7916 & 0.3876 & 0.7142 & 0.2142 & 0.5586 & 0.9748 & 0.5110 \\
\hline SL & -0.5078 & -0.9206 & 0.2006 & 0.3265 & -0.1542 & 0.2526 & 0.2814 & -0.1258 & -0.4416 & -0.1307 & -0.2835 & 0.1360 & 0.2466 & -0.2415 & 0.3155 \\
\hline TPP & 0.0205 & 0.0487 & -0.2479 & -0.0116 & -0.0897 & 0.4122 & 0.0415 & -0.0164 & -0.0334 & -0.1227 & -0.0598 & 0.2170 & 0.0051 & -0.3049 & -0.0447 \\
\hline FLA & 0.8236 & 0.4464 & -0.0651 & -0.8954 & -1.2172 & 0.1725 & 0.1421 & -0.0276 & -0.8125 & -0.5734 & -0.0484 & -0.4723 & 0.2592 & -0.7022 & -0.6406 \\
\hline RWC & 0.7841 & 0.1599 & 0.3830 & 0.9233 & 1.0585 & -0.8315 & -0.7792 & 0.5497 & 1.6283 & 1.6349 & 0.8686 & 0.5339 & -0.7528 & 0.9682 & 0.5282 \\
\hline DFF & -1.7164 & 0.9935 & 0.1975 & 0.4963 & 1.3956 & -1.9151 & -2.1599 & 1.0007 & 2.1703 & 0.1684 & -0.4929 & 0.5822 & -1.0463 & 0.7462 & -0.0119 \\
\hline CT & -0.0322 & -0.0733 & -0.0445 & -0.0271 & -0.1956 & 0.1429 & 0.2657 & 0.0161 & -0.1190 & -0.1232 & -0.0790 & -0.1013 & 0.2475 & -0.8714 & -0.1117 \\
\hline EL & -0.0483 & 0.0332 & 0.0177 & 0.0053 & 0.1397 & -0.7671 & 0.0163 & 0.2689 & -0.0835 & 0.0587 & -0.0553 & -0.0419 & -0.3141 & -0.4803 & -0.0659 \\
\hline GPS & 0.0477 & 0.0398 & 0.0124 & 0.0536 & 0.1417 & -0.3498 & -0.0412 & -0.0286 & 0.0921 & 0.1229 & -0.0067 & 0.3522 & -0.4342 & 0.7049 & 0.4156 \\
\hline SF & -0.1250 & -0.0631 & -0.2439 & -0.2025 & -0.7611 & 0.3207 & 0.2286 & -0.1075 & -0.6574 & -0.4928 & -0.1759 & -0.1284 & 0.1485 & -0.2732 & -0.1709 \\
\hline CHL & 0.4214 & 0.6429 & 0.5581 & 0.0804 & 1.0249 & 0.2842 & -0.6882 & -0.476 & -0.1681 & 1.2963 & 0.8315 & 0.5110 & 0.3966 & 0.9463 & 0.4161 \\
\hline TGW & -0.0168 & 0.0159 & 0.0034 & -0.0405 & -0.0604 & 0.0174 & 0.0457 & 0.0186 & -0.0679 & -0.0312 & -0.0264 & $\mathbf{- 0 . 1 1 9 7}$ & 0.5191 & -0.0511 & -0.0396 \\
\hline DM & 1.5564 & -0.5429 & -0.0462 & -0.4175 & -1.5982 & 1.2646 & 1.2476 & -0.9532 & -0.8342 & -0.2210 & 0.8908 & -0.3583 & 0.9471 & -0.4450 & -0.1393 \\
\hline HI & -1.5435 & -0.7205 & -0.6018 & -0.4413 & -1.4974 & 0.7933 & 1.9639 & 0.9088 & -1.6191 & -1.6878 & -1.9242 & -1.3103 & 0.2030 & -0.8446 & -1.5445 \\
\hline $\mathrm{ZnC}$ & 0.1241 & -0.5281 & 0.3084 & 0.7844 & 0.8526 & 0.2451 & -0.7185 & -0.4185 & 0.2886 & 0.5926 & 0.4071 & 0.5646 & -0.3059 & 0.8668 & 0.8086 \\
\hline GY & 0.6162 & 0.2923 & 0.3055 & 0.6299 & 0.6044 & 0.6953 & -0.3395 & $\mathbf{0 . 3 3 4 7}$ & 0.1342 & 0.8786 & 0.5601 & 0.5789 & 0.6780 & 0.9930 & 0.2259 \\
\hline
\end{tabular}




\section{Rabiya et al.}

altogether leads to the establishment of a positive association between them. A positive direct effect of grain zinc content on grain yield per plant and its indirect effect via no. of tillers per plant $(0.0256)$, relative water content $(0.0237)$, ear length $(0.0144)$, harvest index (0.0654) and spike fertility (0.0531) contributed towards the establishment of positive phenotypic correlation (0.2229) with grain yield/plant. Plant height had its direct effect in a negative direction $(-0.0663)$ resulted in the establishment of its negative correlation with grain yield per plant $(-0.0560)$ was build-up due to negative as well as the endowment of its indirect effect via canopy temperature (-0.0114), ear length $(-0.0143)$, relative water content $(-0.0246)$ and days to maturity (-0.0192). Flag leaf area showed positive phenotypic correlation with grain yield per plant (0.1096) ascribed to its positive direct effect (0.0056) and also substantial indirect effect via days to maturity (0.0227), harvest index (0.0111), spike fertility (0.0503), relative water content (0.0395) and grain zinc content (0.0551). Relative water content exhibited a positive direct effect on grain yield per plant $(0.1533)$ and an indirect effect via seedling length $(0.0181)$, harvest index (0.0478), no. of tiller per plant (0.0133), spike fertility (0.0669), days to maturity (0.0428), and grain zinc content (0.0204) leads to the establishment of positive phenotypic correlation with grain yield $(0.2960)$. Days to $50 \%$ flowering had a positive direct effect on grain yield/plant (0.1413). However, it showed a negative phenotypic correlation with grain yield $(-0.0845)$, signified that it was due to its indirect effect on grain yield via seedling length (0.0444), days to maturity (-0.1559), relative water content $(-0.0320)$ and harvest index (-0.0216). Negative phenotypic correlation of canopy temperature with grain yield per plant $(-0.1521)$ was attributed to its indirect effect via seedling length $(-0.0364)$, no. of tiller/plant (-0.0242), spike fertility $(-0.0425)$, days to maturity $(-0.0747)$, relative water content ($0.0369)$, and grain zinc content (-0.0376) and harvest index (-0.0679), contrarily, it showed a positive direct effect on grain yield/plant (0.1045). The correlation for Ear length found negative with grain yield/plant $(-0.0900)$ was contributed by negative direct effect $(-0.1422)$ and a considerable indirect effect via days to 50 per cent flowering ($0.0236)$, canopy temperature $(-0.0015)$, no. of grains/spike (-0.0020) and grain zinc content ($0.0134)$. The direct positive effect of number of grain per spike on grain yield/plant (0.0228) along with its indirect effect via seedling length (0.0215), ear length (0.0123), relative water content (0.0666), spike fertility (0.0582), harvest index (0.0236) and days to maturity (0.0241) leads to the establishment of positive phenotypic correlation with grain yield (0.1604). Chlorophyll content had its direct effect in the negative direction on grain yield/plant (0.0626 ), whereas it displayed a positive phenotypic association with grain yield/plant $(0.1105)$, which was build-up by its indirect effect in a positive direction via seedling length (0.0354), number of tiller per plant (0.0205), spike fertility (0.0370), relative water content $(0.0356)$, harvest index (0.0507), and grain zinc content (0.0133). A positive indirect effect of thousand-grain weight via grain zinc content $(0.0423)$, relative water content (0.0273), spike fertility (0.0164), days to maturity (0.0171) and harvest index (0.0571) leads to the establishment of Positive phenotypic correlation with grain yield/plant $(0.0530)$. It had its direct effect in a negative direction on grain yield/plant. Days to maturity exhibited negative phenotypic correlation with grain yield/plant (-0.1377), which was contributed by its negative direct effect on grain yield $(-0.1822)$ and also depicted considerable negative indirect effect via seedling length ($0.0438)$, harvest index $(-0.0215)$, spike fertility ($0.0188)$, relative water content $(-0.0360)$ and number of tiller per plant $(-0.0100)$.

\section{Genotypic Path Coefficient}

A positive genotypic correlation was recorded for chlorophyll content with grain yield/plant (0.5601), which was established by a positive direct effect (0.8315). Also, it depicted considerable indirect effect via grain zinc content (0.4071), plant height $(0.7142)$, relative water content $(0.8686)$ and days to maturity (0.8908). A high positive direct effect of Days to maturity on grain yield (0.9471) and its considerable indirect effect via seedling length (0.2466), plant height (0.5586), canopy temperature (0.2475), chlorophyll content (0.3966), flag leaf area (0.2592), test weight $(0.5191)$, spike fertility $(0.1485)$ and harvest index (0.2030). Grain zinc content exhibited high positive direct effect on grain yield (0.8086) and an indirect effect via plant height $(0.5110)$, relative water content $(0.5282)$, seedling length (0.3155), no. of grain/spike 122

Environment Conservation Journal 
(0.4156) and chlorophyll content (0.4161) leads to the establishment of positive genotypic correlation with grain yield. Harvest index exhibited a positive association with grain yield $(0.9930)$, which was attributed to an indirect effect of harvest index on grain yield via the number of grain/spike (0.7049), plant height $(0.9748)$, days to $50 \%$ flowering (0.7462), chlorophyll content $(0.9463)$, relative water content $(0.9682)$ and grain zinc content (0.8668). However, it showed a direct negative effect on grain yield. A positive direct effect of Plant height (0.8284) on grain yield plant leads to establishing a positive correlation $(0.6162)$ between them. It was also depicted by a cumulative indirect effect of plant height on grain yield/plant via flag leaf area (0.8236), no. of tiller per plant (0.0205), no. of grains/spike (0.0477), days to maturity (1.5564), relative water content $(0.7841)$, chlorophyll content $(0.4214)$ and grain zinc content (0.1241). Although seedling length exhibited its direct effect on grain yield $(-0.9206)$ in negative direction, it showed a positive genotypic correlation with grain yield/plant $(0.2923)$ which was caused by its cumulative positive indirect effect on grain yield via plant height $(0.7605)$, relative water content (0.1599), no. of tiller/plant (0.0487), flag leaf area (0.4464), days to $50 \%$ flowering $(0.9935)$, ear length (0.0332), no. of grains/spike (0.0398), chlorophyll content (0.6429) and test weight (0.0159). Positive genotypic association of total number of tillers/plant $(0.3055)$ was ascribed to positive indirect effect via seedling length (0.2006), days to $50 \%$ flowering $(0.1975)$, ear length (0.0177), relative water content (0.3830), chlorophyll content (0.5581) and grain zinc content (0.3084), contrarily, it displayed direct negative effect on grain yield/plant. Flag leaf area exhibited a direct effect on grain yield/plant $(-0.8954)$ in a negative direction. However, it displayed positive genotypic correlation with grain yield (0.6299) contributed by indirect positive effect of flag leaf area via seedling length $(0.3265)$, relative water content (0.9233), days to $50 \%$ flowering (0.4963), no. of grain/spike (0.0563), chlorophyll content $(0.0804)$ and grain zinc content $(0.7844)$. Positive direct effect (1.0585) of relative water content and a substantial indirect effect via plant height (1.5652), days to $50 \%$ flowering (1.3956), ear length (0.1397), number of grain/spike (0.1417), chlorophyll content (1.0249) and grain zinc content
(0.8526) on grain yield/plant leads to the establishment of Positive genotypic association (0.6044). Days to $50 \%$ flowering had direct negative effect on grain yield/plant (-1.9151), but displayed positive genotypic association with grain yield (0.6953) which was build-up by indirect effect via plant height $(0.6533)$, seedling length (0.2526), no. of tiller/plant (0.4122), flag leaf area (0.1725), canopy temperature $(0.1429)$, spike fertility (0.3207), chlorophyll content (0.2842), 1000-grain weight $(0.0174)$, days to maturity (1.2646), HI (0.7933) and grain zinc content (0.2451). Canopy temperature had a positive direct effect on grain yield/plant (0.2657). However, negative correlation with grain yield $(-0.3395)$ has resulted from endowment of substantial indirect effect via plant height $(-0.1853)$, relative water content $(-0.7792)$, days to $50 \%$ flowering ($2.1599)$, no. of grain/spike (-0.0412), chlorophyll content (-0.6882) and grain zinc content $(-0.7185)$. Positive direct effect $(0.2689)$ of ear length and a substantial indirect effect via relative water content (0.5497), days to $50 \%$ flowering (1.0007), canopy temperature $(0.0161)$, test weight $(0.0186)$ and $\mathrm{HI}$ $(0.9088)$ on grain yield/plant leads to the establishment of Positive genotypic correlation (0.3347). A positive direct effect of number of grain/spike (0.0921) and its considerable indirect effect via grain zinc content $(0.2886)$, plant height (0.7916), relative water content (1.6283) and days to $50 \%$ flowering (2.1703) on grain yield leads to the establishment of positive genotypic correlation with it. At the genotypic level, Spike fertility exhibited a high correlation with grain yield (0.8786). However, it had a direct effect in the negative direction on grain yield $(-0.4928)$. The obtained result was due to considerable indirect effect of spike fertility via plant height (0.3876), relative water content (1.6349), days to $50 \%$ flowering (0.1684), ear length (0.0587), no. of grain/spike (0.1229), chlorophyll content (1.2963) and grain zinc content (0.5926). The association for thousand-grain weight was found positive with grain yield (0.5789). However, it directly affected the negative direction on grain yield/plant (0.1197). This result of genotypic path coefficient analysis was obtained due to its considerable indirect effect in positive direction via plant height (0.2142), seedling length $(0.1360)$, no. of tiller/plant (0.2170), days to 50 per cent flowering 
(0.5822), no. of grain/spike (0.3522), relative water content (0.5339), chlorophyll content (0.5110) and grain zinc content $(0.5646)$.

\section{Conclusion}

It can be inferred from the results of correlation coefficient and path coefficient analysis that under timely sowing condition the traits like spike fertility, relative water content, harvest index, flag leaf area, chlorophyll content exhibited strong positive association with the grain yield, positive inter-correlation among themselves and high indirect effect of most of the traits via these traits on grain yield. So, the selection of these traits would be effective and reliable. The traits like spike fertility, harvest index and relative water content

\section{References}

Bahar, B., Yildirim, M. and Yucel, C. 2011. Heat and drought resistance criteria in spring bread wheat (Triticum aestivum L.): Morpho-physiological parameters for heat tolerance. Scientific Research and Essays, 6(10): 2212-2220.

Barman, M., Choudhary, V. K., Singh, S. K., Parveen, R. and Gowda, A. K. 2020b. Correlation and Path Coefficient Analysis in Bread Wheat (Triticum aestivum L.) Genotypes for Morpho-physiological Traits along with Grain $\mathrm{Fe}$ and $\mathrm{Zn}$ Content. Current Journal of Applied Science and Technology, 39(36): 130-140.

Barman, M., Choudhary, V. K., Singh, S. K., Singh, M. K. and Parveen, R. 2020a. Genetic Variability Analysis in Bread Wheat (Triticum aestivum L.) Genotypes for Morpho Physiological Characters and Grain Micronutrient Content. International Research Journal of Pure \& Applied Chemistry, 21(22): 1-8.

Baye, A., Berihun, B., Bantayehu, M. and Derebe, B. 2020. Genotypic and Phenotypic Correlation and Path coefficient analysis for yield and yield-related traits in advanced bread wheat (Triticum aestivum L.) lines. Cogent Food and Agriculture, 6 (1): 1752603.

Cakmak, I. 2008. Enrichment of cereal grains with zinc: agronomic or genetic biofortification? Plant and Soil, 302:1-17.

Chimdesa, O., Mohammed, W. and Eticha, F. 2017. Analysis of genetic variability among bread wheat (Triticum aestivum 1.) genotypes for growth, yield and yield components in Bore district, Oromia regional state. Agriculture, Forestry and Fisheries, 6(6): 188.

Dewey, D. R. and Lu, K. 1959. A Correlation and Path Coefficient Analysis of Components of Crested must be prioritized in the selection process for increased yield because they have a positive association with grain yield. Harvest index has a high direct impact on grain yield, and all other characters indirectly contribute to grain yield through these characters. As a result, selection based on these characters will be more beneficial for improving yield in bread wheat under early heat stress condition.

\section{Acknowledgements}

We are grateful to PG College of Agriculture, DRPCAU, Pusa, Samastipur, Bihar, India and ICRISAT, Hyderabad, India for supporting part of this research and providing facilities for micronutrient analysis.

Wheatgrass Seed Production. Agronomy journal, 51(9): 515-518.

Dogan, R. 2009. The correlation and path coefficient analysis for yield and some yield components of durum wheat (Triticum turgidum L. var. durum) in West Anatolia conditions. Pakistan Journal of Botany, 41(3): 1081-1089.

FAOSTAT. 2019. Crop production. www.fao.org/faostat/. Accessed on January 31, 2021.

Iftikhar, R., Khaliq, I., Ijaz, M. and Rashid, M. R. 2012. Association Analysis of Grain Yield and its Components in Spring Wheat (Triticum aestivum L). American-Eurasian Journal of Agriculture and Environmental Science, 12(3): 389-392.

Joshi, A. K., Mishra, B., Chatrath, R., Ferrara, G. O. and Singh, R. P. 2007. Wheat improvement in India: present status, emerging challenges and future prospects. Euphytica, 157(3): 431-446.

Khan, M. H. and Dar, A. 2010. Correlation and path coefficient analysis of some quantitative traits in wheat. African Crop Science Journal, 18: 9-14.

Kotal, B. D., Das, A. and Choudhury, B. K. 2010. Genetic Variability and Association of Characters in Wheat (Triticum aestivum L.). Asian Journal of Crop Science, 2 (3): 155-160.

Kumar, R., Bhushan, B., Pal, R. and Gaurav, S. S. 2014. Correlation and Path Coefficient Analysis for Quantitative Traits in Wheat (Triticum aestivum L.) under Normal Condition. Annals of Agri-Bio Research, 19 (3): 447-450.

Mohsen, A. A., Hegazy, S. R. and Taha, M. H. 2012. Genotypic and phenotypic interrelationships among yield 


\section{Character association studies in Bread wheat genotypes}

and yield components in Egyptian bread wheat genotypes. Journal of Plant Breeding and Crop Science, 4(1): 9-16.

Sharma, D., Jaiswal, J. P., Singh, N. K., Chauhan, A. and Gahtyari, N. C. 2018. Developing a Selection Criterion for Terminal Heat Tolerance in Bread Wheat Based on Various Mopho-Physiological Traits. International Journal of Current Microbiology and Applied Sciences, 7(7): 2716-2726.

Sharma, P., Kamboj, M. C., Singh, N., Chand, M. and Yadava, R. K. 2018. Path Coefficient and Correlation Studies of Yield and Yield Associated Traits in Advanced Homozygous Lines of Bread Wheat Germplasm. International Journal of Current Microbiology and Applied Science, 7(2): 51-63.

Sharma, V., Kumar, A. and Kumari, S. 2018. Correlation matrix wheat (Triticum asetivum L.) grain zinc $(\mathrm{Zn})$ \& iron $(\mathrm{Fe})$ and among yield contributing traits quantitative and quality traits. Journal of Pharmacognosy and Phytochemistry, 7(4): 329-332.
Stein, A. J. 2010. Global impacts of human mineral malnutrition. Plant and Soil, 335: 133-154.

Verma, S. P., Pathak, V. N. and Verma, O. P. 2019. Interrelationship between Yield and its Contributing Traits in Wheat (Triticum aestivum L). International Journal of Current Microbiology and Applied Science, 8(2): 320932151.

Zafarnaderi, N., Aharizad, S. and Mohammadi, S. A. 2013. Relationship between grain yield and related agronomic traits in bread wheat recombinant inbred lines under water deficit condition. Annals of Biological Research, 4(4): 711. 